A\&A 381, 771-782 (2002)

DOI: $10.1051 / 0004-6361: 20011566$

(c) ESO 2002

\title{
Environmental effects in galaxies
}

\section{Molecular gas, star formation, and activity ${ }^{\star}$}

\author{
D. F. de Mello ${ }^{1}$, T. Wiklind ${ }^{1}$, and M. A. G. Maia ${ }^{2}$ \\ 1 Onsala Space Observatory, 43992 Onsala, Sweden \\ e-mail: duilia@oso.chalmers.se, tommy@oso.chalmers.se \\ 2 Observatório Nacional, Rua Gal. José Cristino 77, RJ 20921, Brazil \\ e-mail: maia@on.br
}

Received 29 December 2000 / Accepted 15 October 2001

\begin{abstract}
In order to study whether there is any correlation between nuclear activities, gas content, and the environment where galaxies reside, we have obtained optical and millimetric spectra for a well-defined sample of intermediate Hubble type spirals in dense environments and in the field. We found that these spirals in dense environments have on average: less molecular gas per blue luminosity, a higher atomic gas fraction, lower current star formation rate, and the same star formation efficiency as field galaxies. Although none of these results stands out as a single strong diagnostic given their statistical significance, taken together they indicate a trend for diminished gas content and star-formation activity in galaxies in high-density environments. Our results suggest that galaxies in dense environments have either (i) consumed their molecular gas via star formation in the past or (ii) that dense environments leads to an inhibition of molecular gas from atomic phase. The similarities in starformation efficiency of the dense environments and field galaxies suggest that the physical processes controling the formation of stars from the molecular gas are local rather than global. We also found that star formation rate per blue luminosity increases linearly as the total amount of gas increases in LINERs. This result, based on a small sample, suggests that LINERs are powered by star formation rather than an AGN.
\end{abstract}

Key words. galaxies: active - galaxies: cluster: general - galaxies: fundamental parameters - galaxies: Seyfert galaxies: spiral

\section{Introduction}

The importance of interactions for triggering activity in galaxies has been extensively explored in the past few decades (e.g. Larson \& Tinsley 1978; Dahari 1984; Kennicutt \& Keel 1984; Keel et al. 1985; Mihos \& Hernquist 1994; Liu \& Kennicutt 1995; Keel 1996). There is no doubt that the environment in which galaxies reside plays a decisive role in galaxy evolution. Nevertheless, there are a few key questions which are still being debated. For instance, the environmental influences on the star formation properties of clusters of galaxies is far from clearcut. Although the molecular gas properties of strongly HI deficient spirals in the Virgo cluster is similar to field spirals (Kenney \& Young 1988), the average star formation

Send offprint requests to: D. de Mello, e-mail: duilia@oso.chalmers.se

* Based on observations at the European Southern Observatory at the $15 \mathrm{~m}$ Swedish ESO Submillimetre telescope, SEST, and at the the $1.52 \mathrm{~m}$ telescope which is operated under the ESO-ON agreement. activity among them are lower than for a sample of field spirals (Kennicutt 1983). This latter effect is, however, not seen in the Coma, Cancer, and A1367 clusters, where star formation activity appears to be enhanced with respect to field spirals (Kennicutt et al. 1984). The Coma spirals are similar to those in the Virgo cluster: deficient in atomic gas, while the molecular gas properties are the same as for field spirals (Casoli et al. 1991, 1996; Gerin \& Casoli 1994). In the Fornax cluster, Horellou et al. (1995) found no evidence for HI deficiency, but an unusual low fraction of molecular gas. For galaxies in loose groups, Maia et al. (1998) find only weak evidence for HI depletion in the early-type spirals.

In compact groups of galaxies the environmental role is also an open issue. Compact groups of galaxies are longlived entities with a space density of galaxies higher than in clusters (see Hickson 1997 for a review). Triggering of star formation through gravitational interaction should therefore be even more important in this environment, but the results indicate differently. Even though 
compact groups present a high fraction of distorted galaxies (Mendes de Oliveira \& Hickson 1994), they do not show an enhancement in far-infrared (FIR) emission (Sulentic \& de Mello Rabaça 1993; Allam et al. 1996), they have low HI content (Williams \& Rood 1987; Huchtmeier 1997), and a normal CO content (Boselli et al. 1996; Leon et al. 1998).

In pairs of galaxies, the scenario is different. The CO and far-infrared luminosities, normalized with either the size of the respective galaxy or $L_{\mathrm{B}}$, is enhanced (Combes et al. 1994). However, whereas the star formation efficiency $(\mathrm{SFE}=$ star formation rate per mass unit of molecular gas) is higher in pairs which are strongly interacting/merging, the average SFE of the whole sample of pairs is similar to normal field spirals. A possible interpretation is that gravitational interactions do not increase the SFE, but increases the amount of star forming gas, possibly through infall of new material.

It has been shown that strong gravitational interactions between galaxies can enhance the star formation rate (SFR) (e.g. Liu \& Kennicutt 1995). It has also been proposed that it is the near environment that mostly affects the evolution of galaxies (Szomoru et al. 1996). However, the connection between the environment, nuclear activities and total gas content has not been able to explain which variables are important in deciding how efficient stars are formed. Are galaxies in dense environments more efficient in forming stars or do they have more fuel? How is the star formation efficiency correlated with the environment where galaxies reside? Is the near environment that mostly affects the evolution of galaxies? An essential step towards answering these open questions is to compare the properties of galaxies in dense environments and in the field.

In this work we present the analysis of the data shown in de Mello et al. (2002, hereafter Paper I) which is a database of molecular and optical spectra of galaxies in dense regions of the Southern sky and in the field.

This paper is organized as follows. Section 2 presents the diagnostics used in this work. Discussion is presented in Sect. 3 and a summary of the main results and conclusions are presented in Sect. 4.

\section{Diagnostics}

Optical and millimetric data have been obtained with the ESO $1.52 \mathrm{~m}$ and the SEST $15 \mathrm{~m}$ radio telescope in La Silla, Chile and we refer to Paper I for further details. Table 1 lists the data as follows. Col. 1: designation in the ESO-Uppsala catalog (Lauberts \& Valentijn 1989, hereafter LV89); Col. 2: type of sample (control sample = CS and high density sample $=\mathrm{HDS}$ ) and morphological type (LV89) $1=\mathrm{Sa}, 2=\mathrm{Sa}-\mathrm{b}, 3=\mathrm{Sb}, 4=\mathrm{Sb}-\mathrm{c}, 5=\mathrm{S} \ldots$, $6=\mathrm{Sc}, \mathrm{Sc}-\mathrm{d}, 7=\mathrm{S}$../Irr, $8=\mathrm{Sd}$; Col. 3: velocity derived from central $\mathrm{CO}(1-0)$ profiles in $\mathrm{km} \mathrm{s}^{-1}$; Col. 4: distance in Mpc corrected for the Virgocentric flow according to model 3.1 in Aaronson et al. (1982); Col. 5: blue luminosity in $L_{\odot}$ derived from $B_{\mathrm{T}}$ magnitude taken from RC3 (de Vaucouleurs et al. 1991); Col. 6: HI masses in $M_{\odot}$ derived using the relation $M_{\mathrm{HI}}=2.36 \times 10^{5} \times D^{2} \times F(\mathrm{HI})$, where $M_{\mathrm{HI}}$ is the $\mathrm{HI}$ mass in $M_{\odot}, D$ is the distance in $\mathrm{Mpc}$, and $F(\mathrm{HI})$ is the $\mathrm{HI}$ flux in $\mathrm{Jy} \mathrm{km} \mathrm{s}^{-1}$ taken from the NASA/IPAC Extragalactic Database (NED) (blank = no HI data available); Col. 7: far-infrared luminosity in $L_{\odot}$ calculated from $L_{\mathrm{FIR}}=5.9 \times 10^{5} D^{2}\left(2.58 \times F_{60}+F_{100}\right)$ where $D$ is the distance in Mpc and $F$ are IRAS fluxes at 60 and $100 \mu \mathrm{m}$ (Moshir et al. 1990); Col. 8: $\mathrm{H}_{2}$ masses in $M_{\odot}$ estimated from the velocity integrated emission, using a $N_{\mathrm{H}_{2}} / I_{\mathrm{CO}}$ conversion ratio of $3 \times 10^{20} \mathrm{~cm}^{-2}$ $\left(\mathrm{K} \mathrm{km} \mathrm{s}^{-1}\right)$; Col. 9: dust temperature in $\mathrm{K}$ calculated as described in Sect. 2.3; Col. 10: dust masses in $M_{\odot}$ calculated as described in Sect. 2.3; and Col. 11: type of activity $(\mathrm{L}=$ LINERs, HII = HII region, blank = no optical data $)$ classified as described in Sect. 2.2. A Hubble constant value of $75 \mathrm{~km} \mathrm{~s}^{-1} \mathrm{Mpc}^{-1}$ was adopted in all calculations.

The diagnostics used in our search for environmental effects are summarized in Tables 2 and 3. Due to the presence of galaxies with higher $L_{\mathrm{B}}$ in the CS (a distance bias in our subsample), masses and luminosities were normalized by $L_{\mathrm{B}}$. Given our morphological selection criteria, we assumed that the mass $/ L_{\mathrm{B}}$ ratio is approximately the same for our galaxies and $L_{\mathrm{B}}$ is thus a measure of the total mass (e.g. Roberts \& Haynes 1994). In Fig. 3a of Paper I we have investigated whether the bias in blue luminosity present in our subsample may cause a bias in our analysis. The correlation found for HDS and CS when we plotted $M_{\mathrm{H}_{2}} / L_{\mathrm{B}}$ as a function of $L_{\mathrm{B}}$ is very similar suggesting no evident bias.

We included in Table 2 the average values given by Leon et al. (1998) for pairs of galaxies, Hickson Compact Groups, starbursts and clusters. We have also included the average values from Leon et al. for galaxies in compact groups using the same morphological criterion we used in our selection ( $\mathrm{Sb}, \mathrm{Sbc}$, and $\mathrm{Sc}$ ).

The distributions of the diagnostics are shown in Fig. 1. The cumulative distributions are shown in Fig. 2. The distribution of morphological types for the HDS and the CS were also included in order to verify how similar the two samples were in terms of morphology. This is a very important aspect to be considered in this type of analysis since morphological appearance is directly correlated with general properties of galaxies. We refer to Sect. 2.4 of Paper I for more details on our morphology selection and to Roberts \& Haynes (1994) for a review on physical parameters along the Hubble sequence.

The significance of the small difference between the mean values of the HDS and the CS was assessed using the Student-t test for unequal variances. The Kolmogorov-Smirnov statistics (KS, hereafter) was used to assess the significance level of the difference between the cumulative distributions. Table 4 shows: Col. 1 the value of $\mathrm{KS}$ (same as $D$ in Press et al. 1989), which is the greatest distance between two cumulative distributions in the KS statistics; Col. 2 the significance level $\mathrm{KS}_{\mathrm{Pb}}$; Col. 3 the Student-t coefficient $T$ for unequal variances; and Col. 4 the significance level $T_{\mathrm{Pb}}$. 
Table 1. The data.

\begin{tabular}{|c|c|c|c|c|c|c|c|c|c|c|}
\hline $\begin{array}{c}\text { ESO-LV } \\
\text { Name } \\
(1)\end{array}$ & $\begin{array}{c}\text { Sample \& } \\
\text { Morph. } \\
(2)\end{array}$ & $\begin{array}{c}V_{\mathrm{CO}} \\
\mathrm{km} \mathrm{s}^{-1} \\
(3) \\
\end{array}$ & $\begin{array}{c}\text { Dist. } \\
\text { Mpc } \\
(4) \\
\end{array}$ & $\begin{array}{c}\log L_{\mathrm{B}} \\
L_{\odot} \\
(5) \\
\end{array}$ & $\begin{array}{c}\log M_{\mathrm{HI}} \\
M_{\odot} \\
(6)\end{array}$ & $\begin{array}{c}L_{\mathrm{FIR}} \times 10^{9} \\
L_{\odot} \\
(7)\end{array}$ & $\begin{array}{c}M_{\mathrm{H}_{2}} \times 10^{9} \\
M_{\odot} \\
(8)\end{array}$ & $\begin{array}{c}T_{\text {dust }} \\
\mathrm{K} \\
(9) \\
\end{array}$ & $\begin{array}{c}M_{\text {dust }} \times 10^{6} \\
M_{\odot} \\
(10)\end{array}$ & $\begin{array}{c}\text { Type of } \\
\text { Activity } \\
(11)\end{array}$ \\
\hline 0310050 & CS 3.5 & 4714 & 59.2 & 10.11 & & $13.60 \pm 0.39$ & $3.30 \pm 0.23$ & $28.64 \pm 0.49$ & $9.98 \pm 0.95$ & HII \\
\hline 1060120 & CS 6 & 4154 & 52.0 & 9.97 & & $6.90 \pm 0.39$ & $1.93 \pm 0.19$ & $29.45 \pm 1.03$ & $4.24 \pm 0.81$ & HII \\
\hline 1080130 & HDS 3.5 & 2941 & 35.8 & 9.78 & & $2.67 \pm 0.15$ & $0.81 \pm 0.07$ & $28.91 \pm 1.00$ & $1.85 \pm 0.35$ & HII \\
\hline 1080200 & CS 3.9 & 1719 & 19.9 & 9.37 & 9.63 & $4.45 \pm 0.20$ & $0.65 \pm 0.03$ & $28.71 \pm 0.82$ & $3.22 \pm 0.49$ & \\
\hline 1190060 & HDS 7.5 & 1256 & 14.4 & 9.48 & 8.91 & $1.66 \pm 0.05$ & $0.11 \pm 0.01$ & $32.62 \pm 0.72$ & $0.55 \pm 0.06$ & \\
\hline 1190190 & HDS 5 & 1527 & 18.0 & 9.94 & 9.14 & $2.52 \pm 0.07$ & $0.42 \pm 0.02$ & $28.85 \pm 0.50$ & $1.77 \pm 0.17$ & HII \\
\hline 1420500 & CS 5 & 2135 & 25.9 & 10.10 & 9.98 & $4.40 \pm 0.09$ & $0.59 \pm 0.04$ & $28.78 \pm 0.44$ & $3.12 \pm 0.24$ & $\mathrm{~L}$ \\
\hline 1460090 & CS 5 & 1652 & 19.0 & 10.131 & 9.47 & $10.40 \pm 0.34$ & $1.10 \pm 0.06$ & $30.88 \pm 0.70$ & $4.75 \pm 0.51$ & HII \\
\hline 1570050 & HDS 5.5 & 1326 & 15.2 & 9.30 & 8.84 & $0.39 \pm 0.02$ & $0.10 \pm 0.01$ & $29.27 \pm 0.96$ & $0.25 \pm 0.05$ & HII \\
\hline 1890070 & CS 4.0 & 3006 & 36.7 & 10.44 & & $9.15 \pm 0.36$ & $1.48 \pm 0.09$ & $31.74 \pm 0.83$ & $3.54 \pm 0.46$ & \\
\hline 2010220 & CS 5 & 3990 & 50.1 & 9.70 & 9.90 & $3.53 \pm 0.23$ & $0.98 \pm 0.07$ & $27.81 \pm 1.03$ & $3.14 \pm 0.67$ & HII \\
\hline 2030180 & CS 4 & 4123 & 52.2 & 10.27 & & $25.39 \pm 1.17$ & $3.30 \pm 0.16$ & $32.28 \pm 1.02$ & $8.88 \pm 1.41$ & HII \\
\hline 2340160 & HDS 5 & 5218 & 66.4 & 10.01 & & $3.72 \pm 0.50$ & $0.94 \pm 0.06$ & $32.22 \pm 3.00$ & $1.32 \pm 0.63$ & HII \\
\hline 2350550 & HDS 5 & 5098 & 64.6 & 10.73 & & $9.92 \pm 1.11$ & $2.04 \pm 0.12$ & $24.89 \pm 1.22$ & $8.61 \pm 6.00$ & $\mathrm{~L}$ \\
\hline 2350570 & HDS 4 & 5069 & 64.2 & 10.03 & & $11.08 \pm 1.30$ & $3.45 \pm 0.13$ & $23.83 \pm 1.23$ & $8.20 \pm 9.77$ & $\mathrm{~L}$ \\
\hline 2370020 & CS 4.5 & 5173 & 65.3 & 10.58 & 10.21 & $13.72 \pm 0.70$ & $4.90 \pm 0.18$ & $27.44 \pm 0.78$ & $3.31 \pm 2.20$ & $\mathrm{~L}$ \\
\hline 2400110 & HDS 4.8 & 2890 & 34.9 & 10.00 & 10.26 & $5.81 \pm 0.31$ & $1.65 \pm 0.05$ & $25.20 \pm 0.93$ & $9.98 \pm 2.16$ & $\mathrm{~L}$ \\
\hline 2400130 & HDS 3 & 3284 & 40.1 & 9.80 & & $5.17 \pm 0.31$ & $1.08 \pm 0.06$ & $27.44 \pm 0.85$ & $5.02 \pm 0.94$ & \\
\hline 2850080 & HDS 4 & 2838 & 35.3 & 10.63 & 10.34 & $4.45 \pm 0$ & $0.64 \pm 0.07$ & $26.69 \pm 0.74$ & $5.18 \pm 0.86$ & $\mathrm{~L}$ \\
\hline 2860820 & HDS 5 & 4958 & 62.8 & 9.98 & & $4.42 \pm 0.51$ & $1.66 \pm 0.09$ & $30.44 \pm 2.23$ & $2.21 \pm 0.87$ & HII \\
\hline 2880260 & HDS 5 & 2383 & 28.8 & 9.79 & 9.57 & $1.42 \pm 0.10$ & $0.32 \pm 0.02$ & $29.40 \pm 1.29$ & $0.88 \pm 0.21$ & $\mathrm{~L}$ \\
\hline 2960380 & CS 4 & 3645 & 45.1 & 9.90 & & $3.73 \pm 0.33$ & $0.44 \pm 0.06$ & $29.33 \pm 1.69$ & $2.35 \pm 0.72$ & HII \\
\hline 3050140 & CS 5 & 4761 & 61.1 & 10.11 & 9.78 & $4.31 \pm 0$ & $2.31 \pm 0.10$ & $32.15 \pm 2.80$ & $1.55 \pm 0.71$ & HII \\
\hline 3470340 & HDS 3 & 1671 & 19.3 & 9.92 & 9.70 & $7.85 \pm 0.79$ & $2.27 \pm 0.06$ & $28.00 \pm 1.54$ & $6.67 \pm 2.14$ & \\
\hline 3500140 & CS 6 & 3400 & 42.0 & 10.09 & 9.73 & $3.30 \pm 0.25$ & $1.23 \pm 0.04$ & $30.04 \pm 1.49$ & $1.79 \pm 0.47$ & HII \\
\hline 3520530 & HDS 3 & 3874 & 48.2 & 10.27 & 9.20 & $21.89 \pm 0.93$ & $6.55 \pm 0.32$ & $30.53 \pm 0.83$ & $0.75 \pm 1.54$ & \\
\hline 3550260 & CS 4 & 1985 & 23.8 & 9.42 & 8.82 & $0.95 \pm 0.07$ & $0.15 \pm 0.02$ & $29.75 \pm 1.46$ & $0.55 \pm 0.14$ & \\
\hline 3550300 & CS 4 & 4448 & 56.1 & 10.25 & 9.82 & $10.05 \pm 0.43$ & $3.33 \pm 0.36$ & $29.04 \pm 0.75$ & $6.75 \pm 0.96$ & $\mathrm{~L}$ \\
\hline 3570190 & HDS 5 & 1789 & 21.4 & 9.83 & 9.43 & $1.52 \pm 0.06$ & $0.41 \pm 0.03$ & $28.48 \pm 0.67$ & $1.15 \pm 0.15$ & HII \\
\hline 4050180 & CS 1 & 3375 & 41.9 & 10.27 & 8.87 & $10.67 \pm 0.68$ & $3.52 \pm 0.17$ & $33.19 \pm 1.50$ & $3.17 \pm 0.70$ & \\
\hline 4060250 & HDS 5 & 1470 & 16.9 & 9.98 & 9.26 & $4.42 \pm 0.20$ & $2.04 \pm 0.05$ & $29.31 \pm 0.83$ & $2.80 \pm 0.43$ & \\
\hline 4060330 & HDS 6 & 1922 & 22.8 & 9.71 & 9.72 & $5.01 \pm 0.21$ & $0.42 \pm 0.02$ & $31.61 \pm 0.90$ & $1.99 \pm 0.29$ & HII \\
\hline 4070140 & CS 5 & 2761 & 33.6 & 9.85 & 9.54 & $3.54 \pm 0.23$ & $0.78 \pm 0.04$ & $31.34 \pm 1.33$ & $1.48 \pm 0.33$ & HII \\
\hline 4190030 & CS 4 & 4146 & 52.8 & 10.20 & 9.82 & $11.19 \pm 0.36$ & $1.10 \pm 0.09$ & $32.48 \pm 0.71$ & $3.77 \pm 0.42$ & HII \\
\hline 4200030 & CS 5 & 4093 & 52.2 & 10.22 & 9.83 & $6.41 \pm 0.41$ & $2.02 \pm 0.14$ & $30.37 \pm 1.24$ & $3.25 \pm 0.70$ & HII \\
\hline 4710200 & CS 4.5 & 3017 & 37.0 & 10.30 & 10.17 & $12.49 \pm 0.53$ & $1.95 \pm 0.13$ & $30.08 \pm 0.81$ & $6.72 \pm 0.96$ & HII \\
\hline 4780060 & CS 4 & 5401 & 68.9 & 10.58 & 9.95 & $51.12 \pm 2.91$ & $10.86 \pm 0.4$ & $32.26 \pm 1.26$ & $7.96 \pm 3.55$ & HII \\
\hline 4820430 & CS 4 & 4073 & 51.9 & 10.17 & 9.74 & $6.57 \pm 0.33$ & $2.35 \pm 0.21$ & $26.86 \pm 0.81$ & $7.33 \pm 1.27$ & HII \\
\hline 4840250 & CS 2 & 4128 & 53.0 & 10.13 & & $16.54 \pm 0.64$ & $2.65 \pm 0.22$ & $32.17 \pm 0.85$ & $5.90 \pm 0.79$ & \\
\hline 5320090 & CS 5 & 2582 & 32.0 & 9.91 & 9.31 & $4.22 \pm 0.20$ & $0.41 \pm 0.05$ & $31.61 \pm 0.98$ & $1.67 \pm 0.26$ & HII \\
\hline 5390050 & CS 5 & 3158 & 39.4 & 9.98 & 8.99 & $5.03 \pm 0.29$ & $1.99 \pm 0.13$ & $30.51 \pm 1.11$ & $2.48 \pm 0.47$ & HII \\
\hline 5450100 & HDS 5 & 1715 & 20.9 & 9.55 & 9.07 & $3.21 \pm 0.14$ & $0.15 \pm 0.01$ & $34.52 \pm 1.08$ & $0.76 \pm 0.11$ & HII \\
\hline 5450110 & HDS 5 & 1456 & 17.5 & 10.35 & 9.60 & $14.78 \pm 0.72$ & $2.15 \pm 0.07$ & $30.79 \pm 0.91$ & $6.88 \pm 1.14$ & \\
\hline 5480070 & HDS 3.5 & 1557 & 19.2 & 9.87 & 9.38 & $1.05 \pm 0.05$ & $0.17 \pm 0.01$ & $26.19 \pm 0.80$ & $1.39 \pm 0.25$ & \\
\hline 5480310 & HDS 3 & 1531 & 18.9 & 9.79 & 8.60 & $3.17 \pm 0.13$ & $0.65 \pm 0.03$ & $28.87 \pm 0.79$ & $2.21 \pm 0.31$ & $\mathrm{~L}$ \\
\hline 5480380 & HDS 6 & 1874 & 23.3 & 10.03 & 9.23 & $10.56 \pm 0.31$ & $0.30 \pm 0.02$ & $41.99 \pm 1.08$ & $0.87 \pm 0.09$ & HII \\
\hline 6010040 & CS 4.6 & 5219 & 66.8 & 10.01 & 9.73 & $3.85 \pm 0.56$ & $1.17 \pm 0.09$ & $28.33 \pm 2.74$ & $3.03 \pm 1.57$ & HII \\
\hline
\end{tabular}


Table 2. Diagnostic quantities.

\begin{tabular}{|c|c|c|c|c|c|c|c|}
\hline Sample $^{\dagger}$ & $\begin{array}{c}\log \left(L_{\mathrm{FIR}} / M_{\mathrm{H}_{2}}\right) \\
L_{\odot} / M_{\odot}\end{array}$ & $\begin{array}{c}\log \left(M_{\mathrm{H}_{2}} / L_{\mathrm{B}}\right) \\
M_{\odot} / L_{\odot}\end{array}$ & $\begin{array}{c}\log \left(L_{\mathrm{FIR}} / L_{\mathrm{B}}\right) \\
L_{\odot} / M_{\odot}\end{array}$ & $\begin{array}{c}\log \left(M_{\mathrm{H}_{2}}+M_{\mathrm{HI}} / L_{\mathrm{B}}\right) \\
M_{\odot} / L_{\odot}\end{array}$ & $\begin{array}{c}\log \left(L_{\mathrm{FIR}} / M_{\mathrm{H}_{2}}+M_{\mathrm{HI}}\right) \\
L_{\odot} / M_{\odot}\end{array}$ & $\begin{array}{c}T_{\mathrm{D}} \\
\mathrm{K}\end{array}$ & $\begin{array}{c}\log \left(M_{\mathrm{D}} / L_{\mathrm{B}}\right) \\
M_{\odot} / L_{\odot}\end{array}$ \\
\hline HDS & $0.74 \pm 0.31$ & $-1.09 \pm 0.39$ & $-0.35 \pm 0.32$ & $-0.35 \pm 0.29$ & $-0.01 \pm 0.42$ & $31.3 \pm 2.8$ & $-3.65 \pm 0.27$ \\
\hline $\mathrm{CS}$ & $0.67 \pm 0.21$ & $-0.91 \pm 0.24$ & $-0.24 \pm 0.22$ & $-0.27 \pm 0.25$ & $0.00 \pm 0.26$ & $30.1 \pm 2.4$ & $-3.48 \pm 0.30$ \\
\hline Pairs & $0.91 \pm 0.43$ & $-0.57 \pm 0.45$ & $0.33 \pm 0.48$ & $-0.47 \pm 0.31$ & $1.04 \pm 0.37$ & $34.9 \pm 6.0$ & $-3.37 \pm 0.40$ \\
\hline $\mathrm{HCG}$ & $0.39 \pm 0.33$ & $-0.61 \pm 0.39$ & $-0.16 \pm 0.45$ & $-0.42 \pm 0.22$ & $-0.02 \pm 0.40$ & $33.1 \pm 5.7$ & $-3.42 \pm 0.36$ \\
\hline $\mathrm{HCG}_{\text {int.type }}$ & $0.37 \pm 0.40$ & $-0.66 \pm 0.35$ & $-0.29 \pm 0.49$ & $-0.14 \pm 0.31$ & $-0.12 \pm 0.40$ & $32.3 \pm 5.6$ & $-3.41 \pm 0.32$ \\
\hline Starbursts & $1.24 \pm 0.39$ & $-0.61 \pm 0.43$ & $0.63 \pm 0.43$ & $-0.36 \pm 0.40$ & $0.91 \pm 0.39$ & $40.4 \pm 6.2$ & $-3.27 \pm 0.34$ \\
\hline Clusters & $0.77 \pm 0.37$ & $-1.08 \pm 0.36$ & $-0.31 \pm 0.40$ & $-0.73 \pm 0.34$ & $0.42 \pm 0.35$ & $33.2 \pm 4.7$ & $-3.78 \pm 0.30$ \\
\hline
\end{tabular}

$\dagger$ HDS is our high density sample, CS is our control sample of isolated galaxies, Pairs, Hickson Compact Groups (HCG), Starbursts and Clusters are from Leon et al. (1998). HCG int.type are HCG galaxies of types Sb, Sbc, and Sc in Leon et al. (1998).
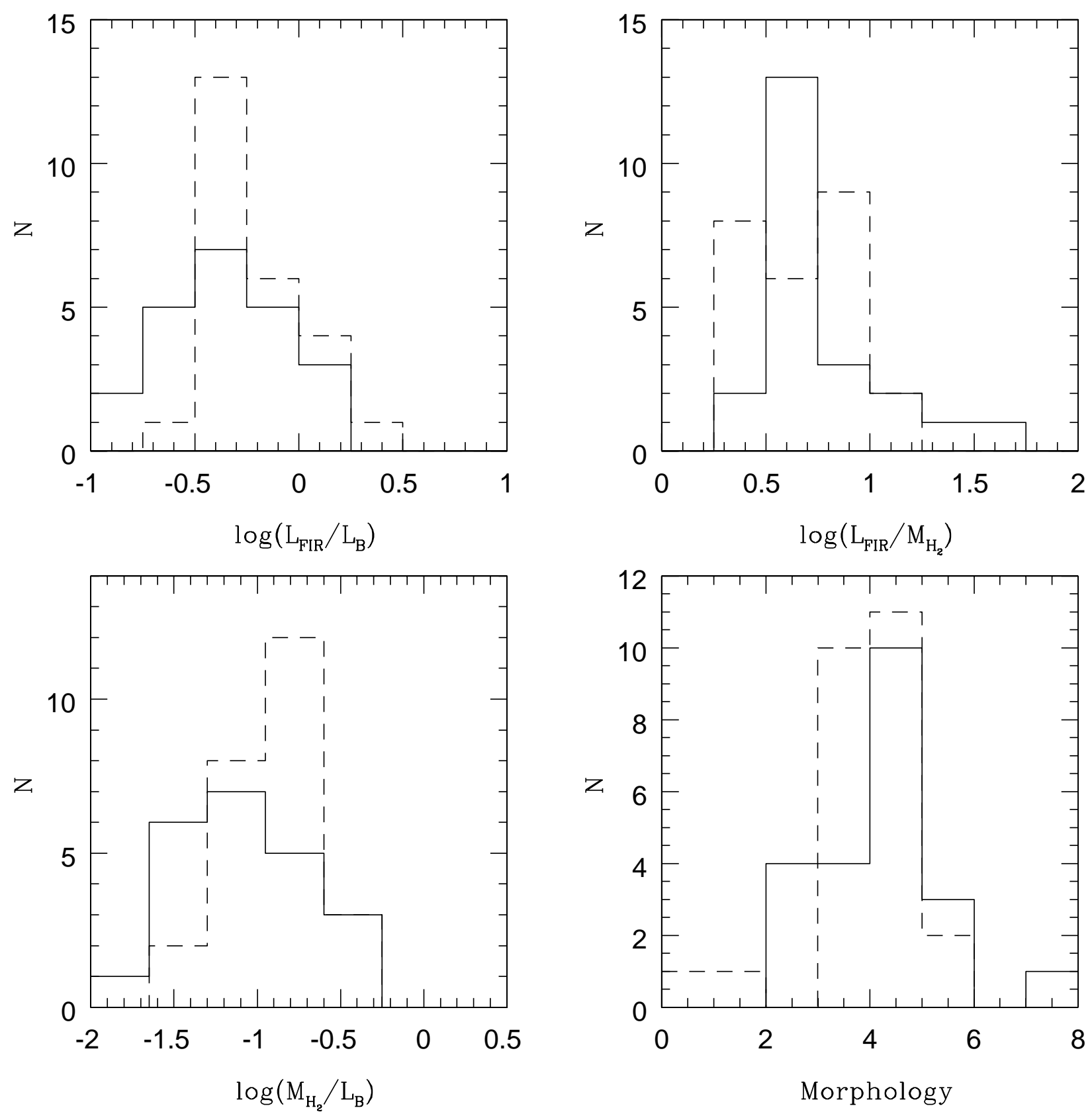

Fig. 1. Upper left panel: distribution of FIR luminosity normalized by blue luminosity; upper right panel: distribution of FIR luminosity normalized by molecular gas; lower left panel: distribution of molecular gas normalized by blue luminosity; lower right panel: distribution of morphological types. Morphological types are: $1=\mathrm{Sa}, 2=\mathrm{Sa}-\mathrm{b}, 3=\mathrm{Sb}, 4=\mathrm{Sb}-\mathrm{c}, 5=\mathrm{S}$..., $6=\mathrm{Sc}$, Sc-d, $7=\mathrm{S} . . /$ Irr, $8=\mathrm{Sd}$. Full line is for the HDS and dashed line is for the CS. Luminosities are in $L_{\odot}$, mass in $M_{\odot}$. 

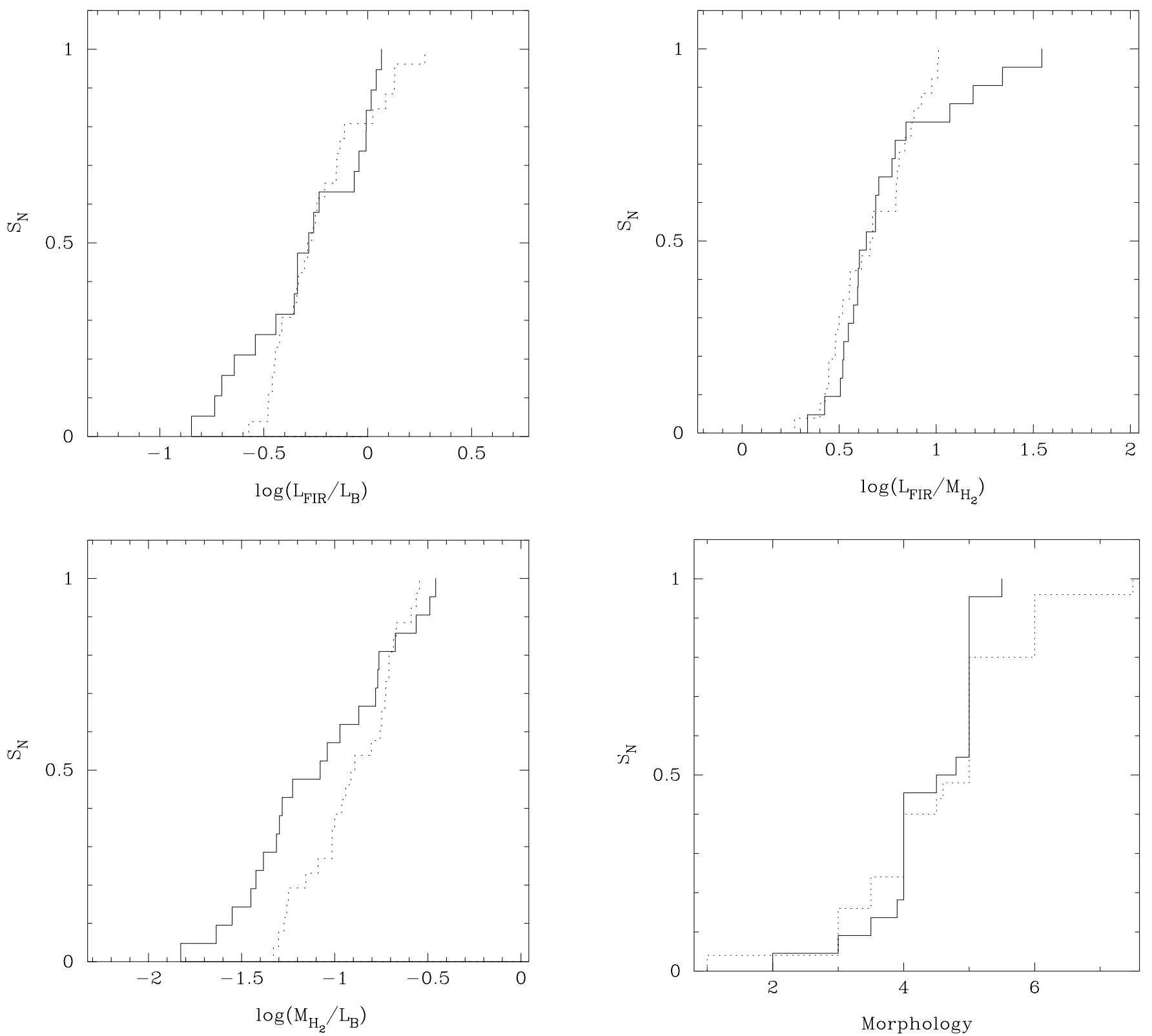

Fig. 2. Cumulative probability distribution of the same parameters of Fig. 1.

Small values of $T_{\mathrm{Pb}}$ indicate that the distributions have significantly different means. Small values of $\mathrm{KS}_{\mathrm{Pb}}$ indicate thatthe cumulative distribution of the HDS is significantly different from that of the CS. The main results are:

- The HDS and the CS have a similar morphology distribution;

- The HDS has on average lower $M_{\mathrm{H}_{2}} / L_{\mathrm{B}}$ than the CS. $M_{\mathrm{H}_{2}} / L_{\mathrm{B}}$ distributions are significantly different. Their means differ at the $93 \%$ level.

Therefore, HDS spirals have overall less molecular gas per blue luminosity than spirals in the field;

- The HDS has on average lower $L_{\mathrm{FIR}} / L_{\mathrm{B}}$ than the CS. $L_{\mathrm{FIR}} / L_{\mathrm{B}}$ distributions are different at the $67 \%$ level. Their means differ at the $84 \%$ level.
Therefore, the HDS spirals have, on average, lower $L_{\mathrm{FIR}} / L_{\mathrm{B}}$ than the $C S$;

- The $L_{\mathrm{FIR}} / M_{\mathrm{H}_{2}}$ ratio can be interpreted as a measure of the star formation efficiency (star formation rate per unit mass of molecular gas). The HDS has on average higher $L_{\mathrm{FIR}} / M_{\mathrm{H}_{2}}$ than the CS. However, the level of significance given by the Stundent-t test and KS statistics is only at the $60 \%$ and $57 \%$ level.

Therefore, the star formation efficiency in the HDS is not statistically different than in the CS.

\subsection{Comparison with other samples}

We showed in Paper I that our subsamples of the HDS and the CS have general properties, such as FIR luminosity, blue luminosity, and molecular gas content, very similar 
Table 3. Correlation coefficients.

\begin{tabular}{lcc}
\hline \multicolumn{1}{c}{ Diagnostic } & $r$ & $r$ \\
& HDS & CS \\
\hline$L_{\mathrm{FIR}} \times M_{\mathrm{H}_{2}}$ & 0.80 & 0.84 \\
$L_{\mathrm{B}} \times M_{\mathrm{H}_{2}}$ & 0.64 & 0.78 \\
$L_{\mathrm{FIR}} / L_{\mathrm{B}} \times M \mathrm{H}_{2} / L_{\mathrm{B}}$ & 0.65 & 0.59 \\
$L_{\mathrm{FIR}} / L_{\mathrm{B}} \times L_{\mathrm{FIR}} / M_{\mathrm{H}_{2}}$ & 0.21 & 0.36 \\
$T_{\mathrm{D}} \times L_{\mathrm{FIR}} / M_{\mathrm{H}_{2}}$ & 0.63 & 0.27 \\
$T_{\mathrm{D}} \times M_{\mathrm{H}_{2}} / M_{\mathrm{D}}$ & 0.56 & 0.44 \\
$L_{\mathrm{FIR}} / L_{\mathrm{B}} \times L_{\mathrm{FIR}} /\left(M_{\mathrm{H}_{2}}+M_{\mathrm{HI}}\right)$ & 0.73 & 0.46 \\
\hline
\end{tabular}

Table 4. Statistical values.

\begin{tabular}{lcccc}
\hline \multicolumn{1}{c}{ Diagnostic } & $\mathrm{KS}$ & $\mathrm{KS}_{\mathrm{Pb}}$ & $T$ & $T_{\mathrm{Pb}}$ \\
\hline Morphology & 0.20 & 0.74 & -0.52 & 0.60 \\
$\log \left(M_{\mathrm{H}_{2}} / L_{\mathrm{B}}\right)$ & 0.33 & 0.16 & -1.89 & 0.07 \\
$\log \left(L_{\mathrm{FIR}} / L_{\mathrm{B}}\right)$ & 0.28 & 0.33 & -1.43 & 0.16 \\
$\log \left(L_{\mathrm{FIR}} / M_{\mathrm{H}_{2}}\right)$ & 0.23 & 0.57 & 0.85 & 0.40 \\
$\log \left(M_{\mathrm{HI}} / L_{\mathrm{B}}\right)$ & 0.31 & 0.42 & 0.45 & 0.65 \\
$\log \left(M_{\mathrm{H}}+M_{\mathrm{HI}} / L_{\mathrm{B}}\right)$ & 0.42 & 0.09 & -1.19 & 0.24 \\
$\log \left(L_{\mathrm{FIR}} / M_{\mathrm{H}_{2}}+M_{\mathrm{HI}}\right)$ & 0.27 & 0.55 & 0.16 & 0.87 \\
$\log \left(M_{\mathrm{HI}} / M_{\mathrm{H}_{2}}\right)$ & 0.40 & 0.12 & 0.87 & 0.39 \\
$T_{\mathrm{D}}$ & 0.28 & 0.33 & -0.63 & 0.54 \\
\hline
\end{tabular}

$\mathrm{KS}$ is the greatest distance between two cumulative distributions in the Kolmogorov-Smirnov statistics. $\mathrm{KS}_{\mathrm{Pb}}$ is the significance level to the null hypothesis that the data sets are drawn from the same distibution. $T$ is the Student $t$ coefficient for unequal variances and $T_{\mathrm{Pb}}$ is the significance level.

to other galaxies such as normal spiral galaxies (Young et al. 1989; Braine et al. 1993), ultraluminous FIR galaxies (Sanders et al. 1991), and galaxies in the Coma and Fornax clusters (Casoli et al. 1991; Horellou et al. 1995); i.e. they are not a separate class of objects.

However, our $M_{\mathrm{H}_{2}} / L_{\mathrm{B}}$ average value $\left(\log \left(M_{\mathrm{H}_{2}} / L_{\mathrm{B}}\right)=\right.$ $-0.91 \pm 0.24$ for the $\mathrm{CS})$ is lower than the classical value from Young \& Knezek (1989) $\left(\log \left(M_{\mathrm{H}_{2}} / L_{\mathrm{B}}\right) \sim-0.77 \pm\right.$ $\left.0.05 M_{\odot} / L_{\odot}\right)$. The main reason for this disagreement is that Young \& Knezek sample of spirals was FIR-selected, it is biased towards higher $L_{\mathrm{FIR}}$ and therefore towards higher $M_{\mathrm{H}_{2}}$. A sample like CS which is not selected with any FIR limit, is more realistic for the field.

Another sample of isolated galaxies such as the one used in Leon et al. (1998) shows a high dispersion of $\log \left(M_{\mathrm{H}_{2}} / L_{\mathrm{B}}\right) \sim-0.78 \pm 0.58 M_{\odot} / L_{\odot}$ which demonstrates that their sample is not very homogeneous in terms of molecular gas content. This is due to the fact that the sample of Leon et al. (1998) includes all morphological types, whereas our sample has only galaxies $\mathrm{Sb}, \mathrm{Sbc}$, and Sc. This is also seen when one compares Hickson Compact Groups of all types with HCG later than Sa and earlier than Sd given in Table 2.

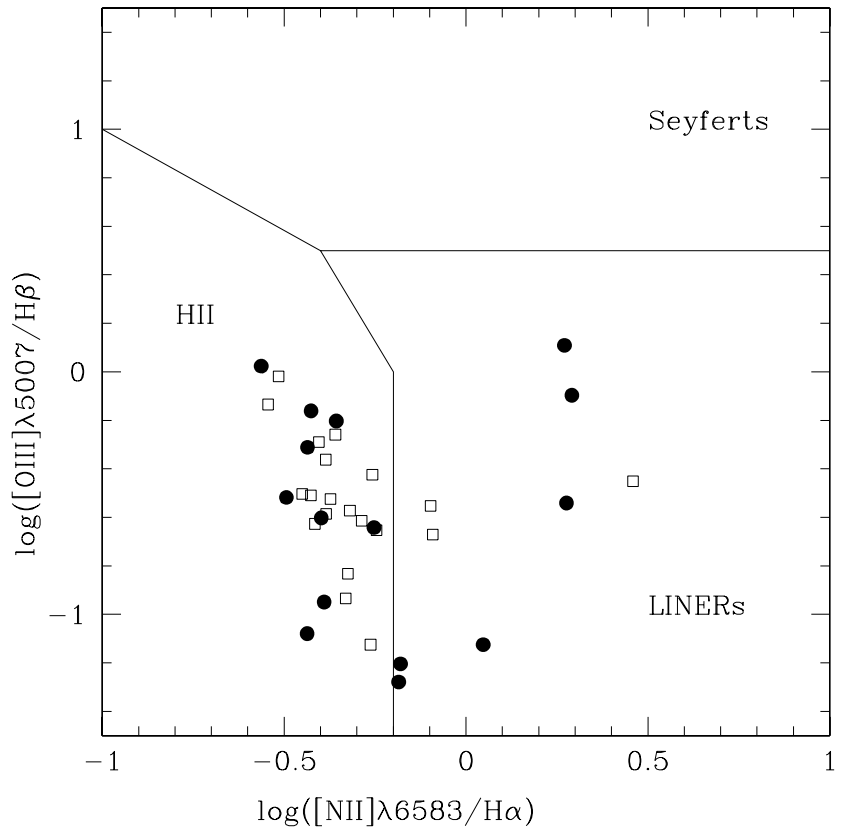

Fig. 3. Diagnostic diagram $-\log ([\mathrm{OIII}] \lambda 5007 / \mathrm{H} \beta)$ versus $\log ([\mathrm{NII}] \lambda 6583 / \mathrm{H} \alpha)$. Solid lines divide the nuclear activities in Seyferts, LINERs, HII galaxies, based on Veilleux \& Osterbrock (1987). The CS is marked by open squares. The HDS is marked by solid circles.

\subsection{Nuclear activity}

It is believed that one of the environmental effects in disk systems is the efficient transport of gas to the centers of the galaxies caused by gravitational interaction. This process can in principle trigger nuclear thermal (starbursts) and nonthermal activities (AGNs). The classification of the type of activity in our sample was done by measuring line-intensity ratios and applying standard diagnostic diagrams (Baldwin et al. 1981; Veilleux \& Osterbrock 1987). Fluxes have been corrected for galactic and internal reddening. However, due to the close wavelength separation of the lines used in the ratios, the internal reddening correction is nearly negligible. In Fig. 3 we show the $\log ([\mathrm{OIII}] \lambda 5007 / \mathrm{H} \beta)$ versus $\log ([\mathrm{NII}] \lambda 6583 / \mathrm{H} \alpha)$ for 35 galaxies (15 in the HDS and 20 in the CS). Most of the galaxies have spectra showing signs of star formation (HII-type). A total of 9 LINERs were identified, 3 in the CS and 6 in the HDS. Field spiral galaxies and close pairs of galaxies are reported in the literature to have $19 \%$ and $10 \%$ of LINERs (Ho et al. 1997; Barton et al. 2000).

The lack of Seyfert galaxies in our subsample seems to be in disagreement with the recent work by Coziol et al. (2000) who reported a high number of Seyfert galaxies in compact groups of galaxies. However, whereas our HDS subsample includes only intermeditate spiral galaxies, their sample includes both early- and late-type galaxies, with the Seyferts being more common in earlier types (E and S0s). However, intermediate type spirals can also host AGNs. For example, in the work by Maiolino et al. (1997) where they studied the molecular gas content of 94 Seyfert galaxies, there are 43 spirals of intermediate 
types. Therefore, the lack of Seyferts in our subsamples could be either due to a bias in our classification of activities or to the size of our small subsample. The fact that we used $\mathrm{H} \alpha / \mathrm{H} \beta=2.86$ in some galaxies where it was not possible to measure $\mathrm{H} \beta$ in emission will not change the the diagnostic diagram significantly. For instance, if we use $\mathrm{H} \alpha / \mathrm{H} \beta=3.1$ (typical of AGNs) instead of $\mathrm{H} \alpha / \mathrm{H} \beta=2.86$, the change in the vertical axis will not be large enough to bring the data points into the Seyfert regions. Therefore, the lack of Seyferts in our subsample can be explained by our morphology selection and by the small number of galaxies in or subsample.

Figure 4 shows the correlation between the total molecular gas, $M_{\mathrm{H}_{2}}$, and $L_{\mathrm{FIR}}$, both divided by $L_{\mathrm{B}}$. The correlation coefficient is 0.65 and 0.59 for the HDS and CS. However, if we consider only LINERs, the correlation coefficient increases to 0.99 and 0.75 ; i.e. the star formation rate (SFR) per blue luminosity increases linearly as the total amount of molecular gas increases in LINERs, in particular for the HDS. This result might have important implications in the understanding of the nature of LINERs (e.g. Alonso-Herrero et al. 2000). However, a larger sample of LINERs should be used in order to statistically test the significance of this trend. Another interesting result is the fact that the galaxies which deviates from the linear correlation are all non-AGN galaxies. Hence, AGN heating of dust cannot be invoked as an explanation for the higher $L_{\mathrm{FIR}} / L_{\mathrm{B}}$ for a given $M_{\mathrm{H}_{2}} / L_{\mathrm{B}}$.

\subsection{Dust temperature}

Dust temperature can provide a better understanding of the physical conditions inside the galaxies. Warm dust $\left(T_{\mathrm{D}}>50 \mathrm{~K}\right)$ is typical of molecular clouds where massive stars $\left(>6 M_{\odot}\right)$ reside, whereas cold dust $\left(T_{\mathrm{D}}<30 \mathrm{~K}\right)$ traces quiescent molecular clouds heated by the interstellar radiation field. However, the limited spatial resolution of IRAS gives an average of extended cold dust emission and small hot emission areas (the dust emissivity goes as $\left.T_{\mathrm{D}}^{4}\right)$. Nevertheless, a higher dust temperature is indicative of current star formation. From the ratio of the IRAS fluxes at $60 \mu \mathrm{m}$ and $100 \mu \mathrm{m}$ we derived dust temperatures assuming $\kappa_{\nu} \propto \nu$. Dust masses were derived using the following equation,

$M_{\mathrm{D}}=4.8 \times 10^{-11} \alpha S_{100} d^{2} /\left(\kappa_{\nu} B_{\nu}\left(T_{\mathrm{D}}\right)\right)$

where $S_{100}$ is the IRAS flux at $100 \mu \mathrm{m}$ in $\mathrm{Jy}, \kappa_{\nu}$ is the mass opacity of the dust $\left(\kappa_{\nu}=25 \mathrm{~cm}^{2} \mathrm{~g}^{-1}\right.$, Hildebrand 1983), $B_{\nu}\left(T_{\mathrm{D}}\right)$ is the Planck function, $d$ is the distance in Mpc, and $\alpha$ the molecular gas-to-dust mass ratio ( $\alpha=700$, Thronson \& Telesco 1986).

The average values of $T_{\mathrm{D}}$ (HDS $=31.3 \pm 2.8$ and $\mathrm{CS}=30.1 \pm 2.4$ ) are typical of spiral galaxies (Sage 1993; Wiklind et al. 1995). In Fig. 5 we see that there is a lack of correlation between SFE and $T_{\mathrm{D}}$ for the CS and a weak correlation for the HDS (correlation coefficients are $\sim 0.3$ and 0.6 , respectively). However, the relatively low values

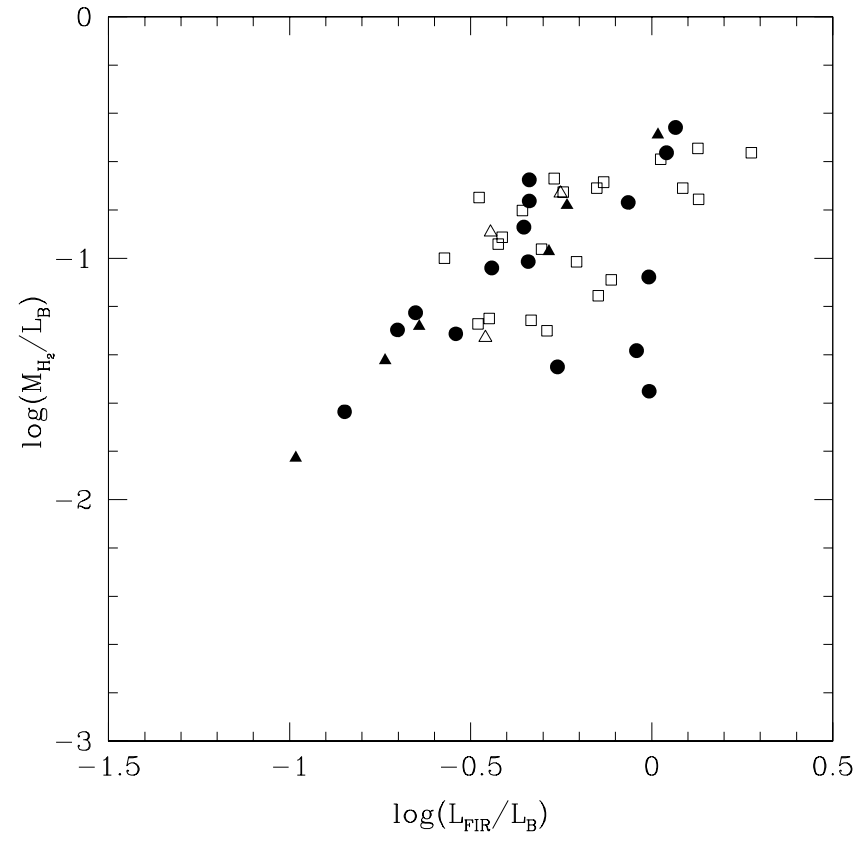

Fig. 4. Molecular gas normalized by blue luminosity as a function of FIR luminosity normalized by blue luminosity. The CS is marked by open symbols and HDS by filled symbols. LINERs are marked by triangles. Luminosities are in $L_{\odot}$ and mass in $M_{\odot}$.

Table 5. $T_{\mathrm{D}}$ and SFE average values.

\begin{tabular}{c|cc|cc}
\hline & \multicolumn{2}{c}{ LINERs } & \multicolumn{2}{c}{ Non-LINERs } \\
Diagnostic & HDS & CS & HDS & CS \\
\hline$T_{\mathrm{D}}$ & $26.5 \pm 2.3$ & $28.4 \pm 0.8$ & $31.3 \pm 2.8$ & $30.1 \pm 2.4$ \\
$\log L_{\mathrm{FIR}} / M_{\mathrm{H}_{2}}$ & $0.65 \pm 0.12$ & $0.60 \pm 0.23$ & $0.77 \pm 0.35$ & $0.68 \pm 0.21$ \\
\hline
\end{tabular}

of $T_{\mathrm{D}}$ for LINERs (Table 5 ) might have important implications regarding the interpretation of the source that powers the nuclear region of these galaxies. A low $T_{\mathrm{D}}$ implies a low current star formation and no powerful black hole. The scenario proposed by Alonso-Herrero et al. (2000) fits well our results. If LINERs are aging starbursts they should have low $T_{\mathrm{D}}$ since their massive stars would have evolved after 5-10 Myr. This is also suggested by the lower averages of $\log L_{\mathrm{FIR}} / M_{\mathrm{H}_{2}}$ for LINERs. However, we cannot exclude the presence of a central black hole with reduced activity (Ji et al. 2000). In view of the small number of LINERs in our sample it is not possible to reach any firm conclusion regarding the correlation between the amount of fueling gas and the starburst and/or AGN activities.

\subsection{Star formation rate}

The $\mathrm{H} \alpha$ equivalent width $(E W)$ is defined as the emissionline luminosity normalized to the adjacent continuum flux, and hence is proportional to the star formation rate per unit (red) luminosity. The mean value of $E W(\mathrm{H} \alpha)$ for the HDS and CS are $14.9 \pm 11.7$ and $13.4 \pm 9.5 \AA$, respectively. These values are lower than the ones found in Pastoriza et al. (1994) for galaxies in other subsamples of HDS and 


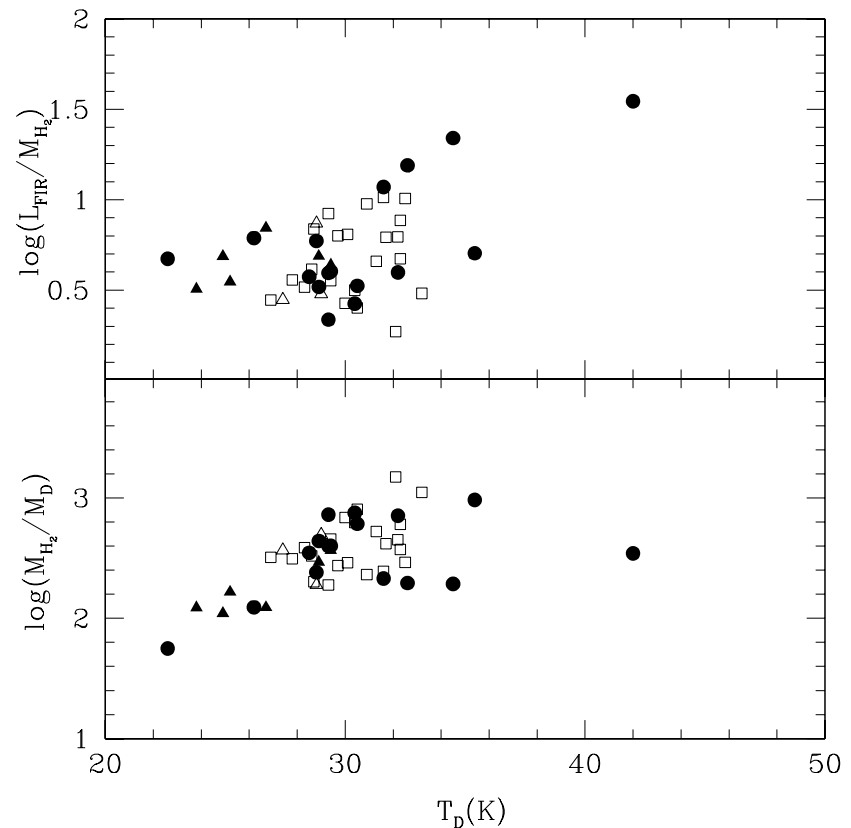

Fig. 5. Upper panel: FIR luminosity normalized by molecular gas as a function of dust temperature in K. Lower panel: molecular gas normalized by dust mass as a function of dust temperature in K. Luminosities are in $L_{\odot}$ and mass in $M_{\odot}$. Symbols are the same as in Fig. 4.

CS galaxies. The main difference between the latter subsample and our subsample is the morphology and type of activity. The majority of the galaxies in Pastoriza et al. (1994) with large $E W_{\mathrm{s}}$ are either later-type spirals or classical Seyfert galaxies, like NGC 7582. In our subsample we have spirals of intermediate type and no Seyferts.

Since the HDS has more LINERs than the CS and LINERs tend to have smaller $E W(\mathrm{H} \alpha)$, we find that, if we disregard all LINERs, the mean value of $E W(\mathrm{H} \alpha)$ changes to $19.8 \pm 10.1$ and $15.3 \pm 9.0 \AA$, for the HDS and CS, respectively. However, the number of galaxies with measured $E W(\mathrm{H} \alpha)$ is too small to give any statistically significant result. SFRs based on $L(\mathrm{H} \alpha)$ can also be severely underestimated due to dust internal to the galaxies (Bushouse 1987; Kennicutt 1998). Therefore, we use $L_{\text {FIR }}$ as a diagnostic of the recent star formation.

\section{Discussion}

\subsection{Environment}

In order to investigate whether there is any correlation between the environment, the type of activity, and the total amount of molecular gas, we have identified galaxies in the HDS which are also part of groups according to Maia et al. (1989). As environmental parameters we used the number of companions and the mean separation between the galaxies of each group $\left(N_{\mathrm{g}}\right.$ and $r_{\mathrm{p}}$ in Maia et al. 1989). We plotted $M_{\mathrm{H}_{2}} / L_{\mathrm{B}}, E W(\mathrm{H} \alpha)$ and $\mathrm{SFE}$, as a function of $N_{\mathrm{g}}$ and $r_{\mathrm{p}}$ (Figs. 6 and 7 ). We also marked in these figures the mean values for clusters of galaxies, starburst galaxies, and Hickson compact groups. No correlation between $M_{\mathrm{H}_{2}} / L_{\mathrm{B}}$

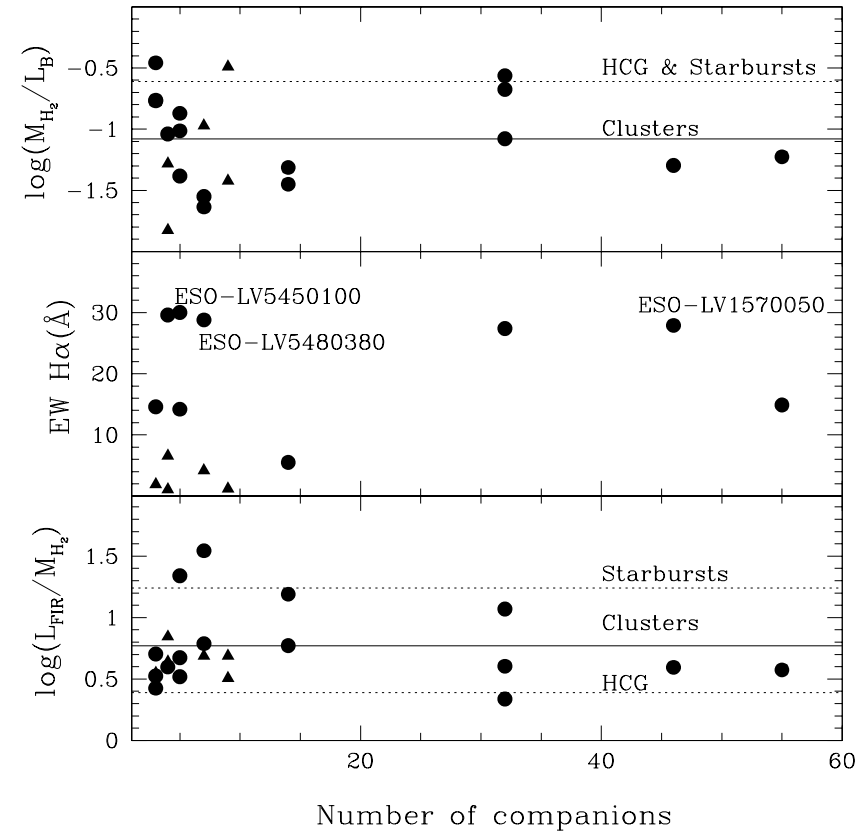

Fig. 6. Upper panel: molecular gas normalized by blue luminosity as a function of the number of companions of galaxies $\left(N_{\mathrm{g}}\right)$ in the HDS. Middle panel: the equivalent width of $\mathrm{H} \alpha$ in $\AA$ as a function of $N_{\mathrm{g}}$ in the HDS. Lower panel: FIR luminosity normalized by molecular gas as a function of $N_{\mathrm{g}}$ in the HDS. Horizontal lines are average values for Hickson compact groups (HCG), starbursts and clusters from Leon et al. (1998). LINERs are marked by triangles. Luminosities are in $L_{\odot}$ and mass in $M_{\odot}$.

and the environmental parameters is found. There are galaxies as gaseous as starbursts and as poor as clusters in all environments. LINERs were found only in groups with less than 20 members but are present in groups with either small or large separations between the members.

We also found no correlation between $E W(\mathrm{H} \alpha)$ and the environmental parameters. This is in disagreement with Barton et al. (2000) who found that $E W(\mathrm{H} \alpha)$ anticorrelates strongly with pairs spatial separation and velocity separation. However, this could be either due to the small size of our sample in comparison with Barton et al. (2000) which contains 502 galaxies, and/or to the differences in the selection criterion imposed by our sample and Barton et al. The latter sample mixes pairs and groups of galaxies whereas our sample has galaxies in dense environments and no isolated pairs of galaxies.

The lack of a trend in Figs. 6 and 7 is clear. However, we decided to check some galaxies individualy. We noticed that three of the HDS galaxies (ESOLV1570050, ESO-LV5450100, and ESO-LV5480380) with the largest values of $E W(\mathrm{H} \alpha)$ have less $M_{\mathrm{H}_{2}} / L_{\mathrm{B}}$ than the average value. They are located in groups with small and large separations (e.g. $r_{\mathrm{pESO}-\mathrm{LV} 5450100}=0.40 \mathrm{Mpc}$, $r_{\mathrm{pESO}-\mathrm{LV} 5480380}=0.94 \mathrm{Mpc}$, and $r_{\mathrm{pESO}-\mathrm{LV} 1570050=}=$ $1.3 \mathrm{Mpc}$ ) and with small and large number of companions (ESO-LV5450100 has only 4 companions, ESO-LV5480380 has 6 companions whereas ESO-LV1570050 has 45 companions). Therefore, for these three galaxies, these 


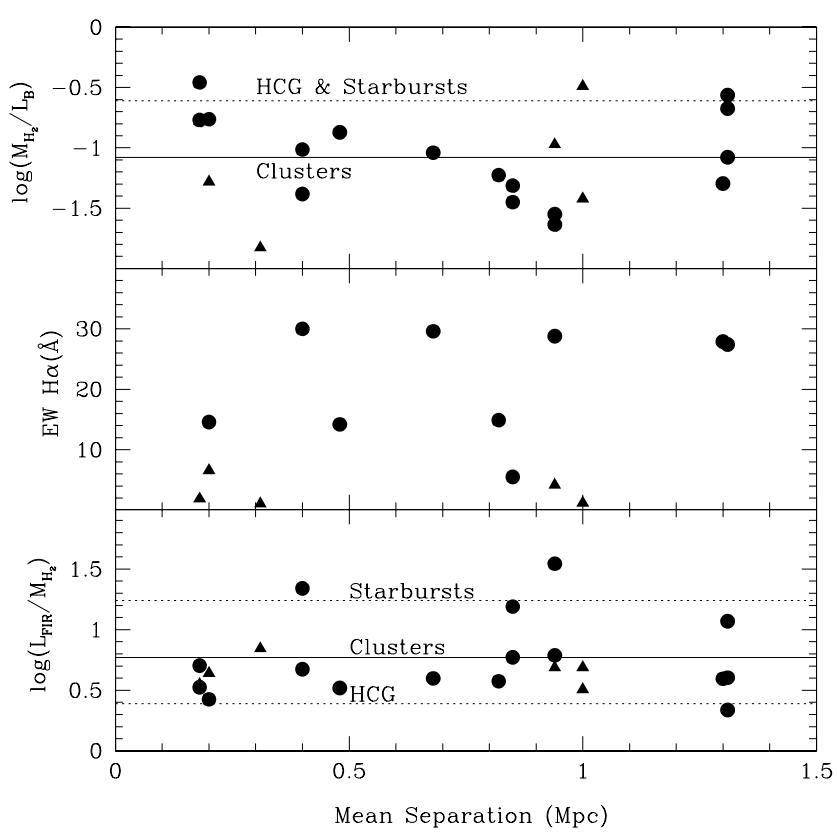

Fig. 7. Upper panel: molecular gas normalized by blue luminosity as a function of the mean separation $\left(r_{\mathrm{p}}\right)$ between galaxies in the HDS. Middle panel: the equivalent width of $\mathrm{H} \alpha$ in $\AA$ as a function of $r_{\mathrm{p}}$ in the HDS. Lower panel: FIR luminosity normalized by molecular gas as a function of $r_{\mathrm{p}}$ in the HDS. Horizontal lines are for Hickson compact groups (HCG), starbursts and clusters from Leon et al. (1998). LINERs are marked by triangles. Luminosities are in $L_{\odot}$, mass in $M_{\odot}$, and $r_{\mathrm{p}}$ in Mpc.

environmental parameters are not responsible for the enhancement of star-formation and defficiency in molecular gas.

There is also no correlation between SFE and the environmental parameters. (Figs. 6 and 7 lower panels). Combes et al. (1994) also found no correlation between SFE and separation in pairs of galaxies. However, because pairs of galaxies are FIR enhanced they suggested that pairs have an increase in the molecular gas content. However, this is not the case for our sample. No clear trend between the environment and $L_{\mathrm{FIR}} / L_{\mathrm{B}}$ is found (Fig. 8). We note that the two galaxies with the largest number of companions have the lowest $L_{\mathrm{FIR}} / L_{\mathrm{B}}$. This could be an indication of the influence of the environment on the star formation rate of these galaxies and should be investigated for a larger sample. Similar results were suggested by Hashimoto et al. (1998) using the [OII] emission line as a diagnostic of SFR.

The possibility of a correlation with the crossing time $\left(t_{v}\right.$ in Maia et al. 1989) was also checked and no correlation was found. Therefore, we conclude that there is no clear correlation between molecular gas content, SFR, SFE and the environmental parameters.

\subsection{Total gas and morphology}

It is possible that interacting galaxies like galaxies in compact groups have less current star formation because they
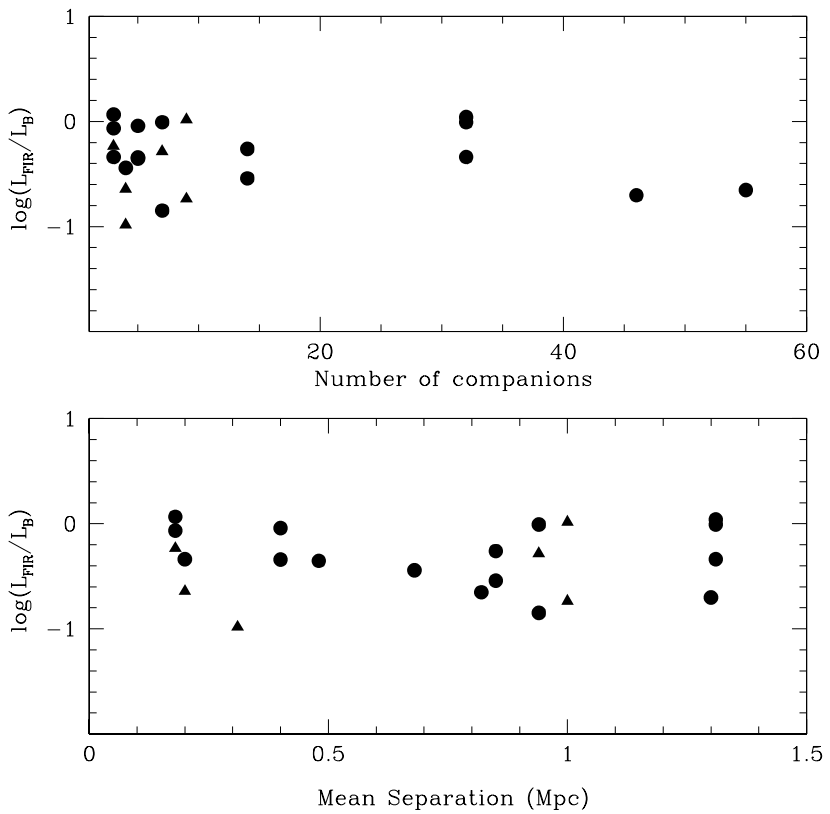

Fig. 8. Upper panel: number of companions $\left(N_{\mathrm{g}}\right)$ of galaxies in the HDS as a function of FIR luminosity normalized by the blue luminosity. Lower panel: Mean separation $\left(r_{\mathrm{p}}\right)$ between the members of the groups in the HDS as a function of FIR luminosity normalized by the blue luminosity. LINERs are marked by triangles. Luminosities are in $L_{\odot}$ and $r_{\mathrm{p}}$ in Mpc.

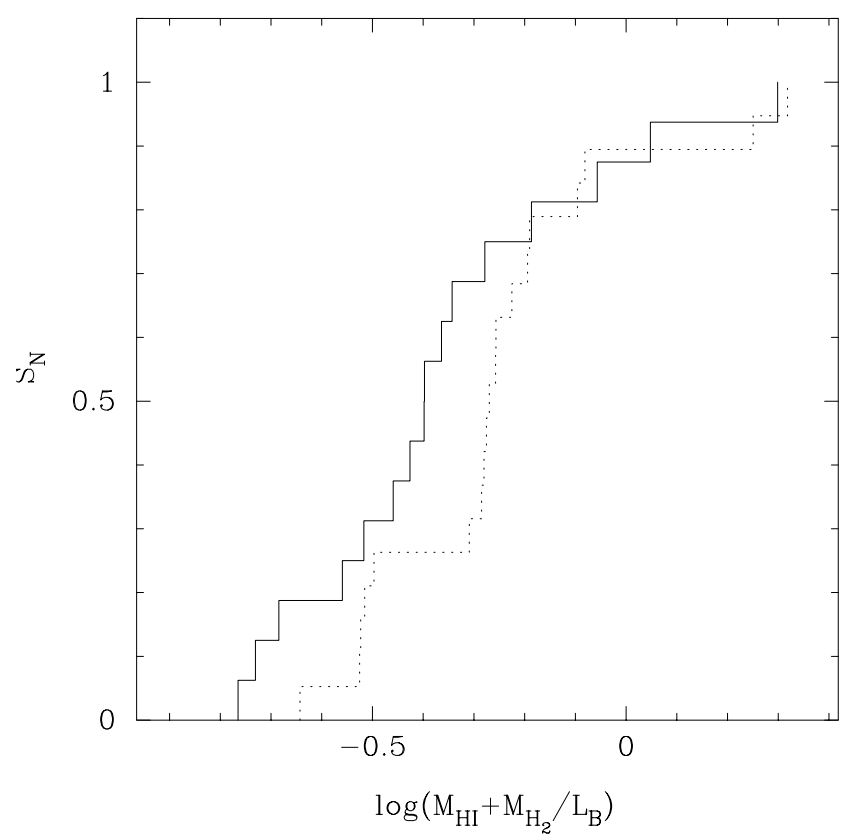

Fig. 9. Cumulative probability distribution of the total gas normalized by total gas. HDS is marked by solid line and CS by dotted line. Luminosity is in $L_{\odot}$ and mass in $M_{\odot}$.

have less total fuel (Sulentic \& de Mello Rabaça 1993; Leon et al. 1998). Therefore, the total amount of gas, $\mathrm{H}_{2}+\mathrm{HI}$, might be a better SFE indicator in very dense environments. In order to check if this is valid for our 


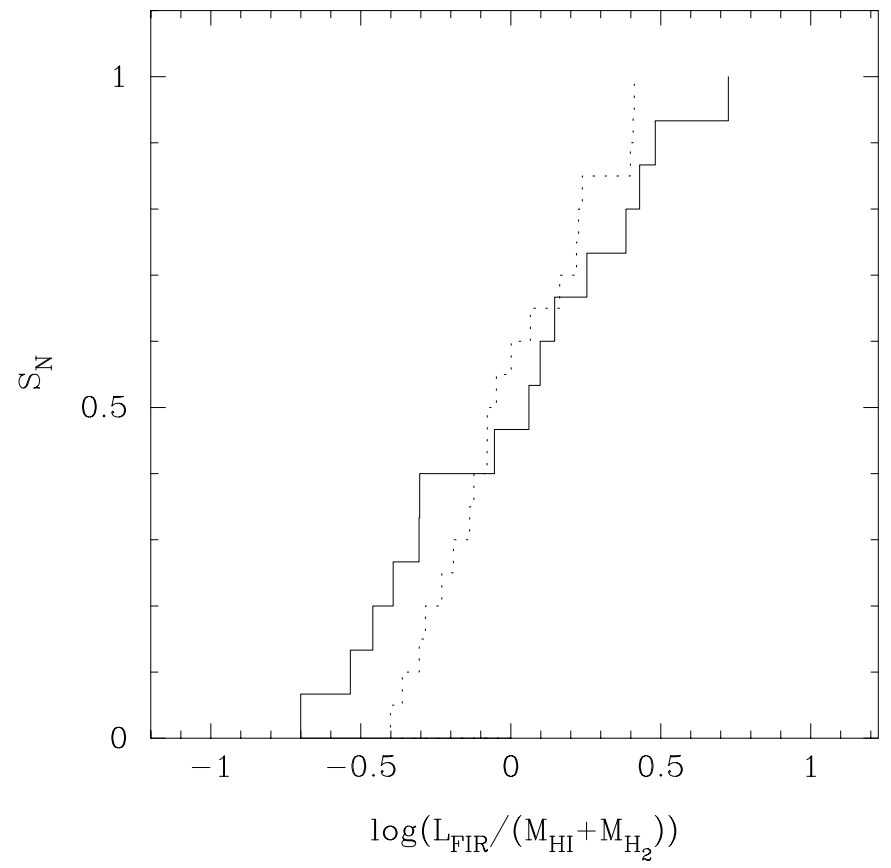

Fig. 10. Cumulative probability distribution of FIR luminosity normalized by total gas. HDS is marked by solid line and CS by dotted line. Luminosity is in $L_{\odot}$ and mass in $M_{\odot}$.

sample, we used the HI data available in the NED for 35 galaxies of our sample. Our results are:

- Total gas: $\left(M_{\mathrm{HI}}+M_{\mathrm{H}_{2}}\right) / L_{\mathrm{B}}$ distributions (Fig. 9) are significantly different (91\% level).

The HDS has lower total gas than the CS;

- Gas fraction: the average values of $\log \left(M_{\mathrm{HI}} / M_{\mathrm{H}_{2}}\right)$ for the HDS and CS are 0.69 \pm 0.59 and $0.51 \pm 0.46$. $M_{\mathrm{HI}} / M_{\mathrm{H}_{2}}$ distributions are significantly different $(88 \%$ level).

The HDS in comparison with CS has, more atomic gas, or a higher atomic gas fraction. Similar values $\left(\log \left(M_{\mathrm{HI}} / M_{\mathrm{H}_{2}}\right)=0.62 \pm 0.43\right)$ are found by Horellou $\&$ Booth (1997) for a sample of southern interacting galaxies;

- SFE ( $\boldsymbol{L}_{\text {FIR }} /$ total gas): the star formation efficiency distributions calculated using the total gas, $L_{\mathrm{FIR}} /\left(M_{\mathrm{HI}}+M_{\mathrm{H}_{2}}\right)$, (Fig. 10) are not significantly different ( $55 \%$ level).

Figure 11 shows the SFE indicators as a function of $L_{\mathrm{FIR}} / L_{\mathrm{B}}$ (both plots include only galaxies for which HI data were available). The large dispersion seen in the upper panel decreases in the lower panel where we include the neutral gas. The correlation coefficient changes from 0.21 to 0.73 in the HDS and 0.59 to 0.46 in the CS ( $r$ in Table 3).

Figure 12 shows the SFE including the atomic gas, $L_{\mathrm{FIR}} /\left(M_{\mathrm{HI}}+M_{\mathrm{H}_{2}}\right)$, as a function of the SFE without the atomic gas. The three most efficient galaxies previously found using only the molecular gas (ESO-LV5480380, ESO-LV5450100, ESO-LV1190060) still have high values of SFE when we include the HI content. However, it is interesting to see how other

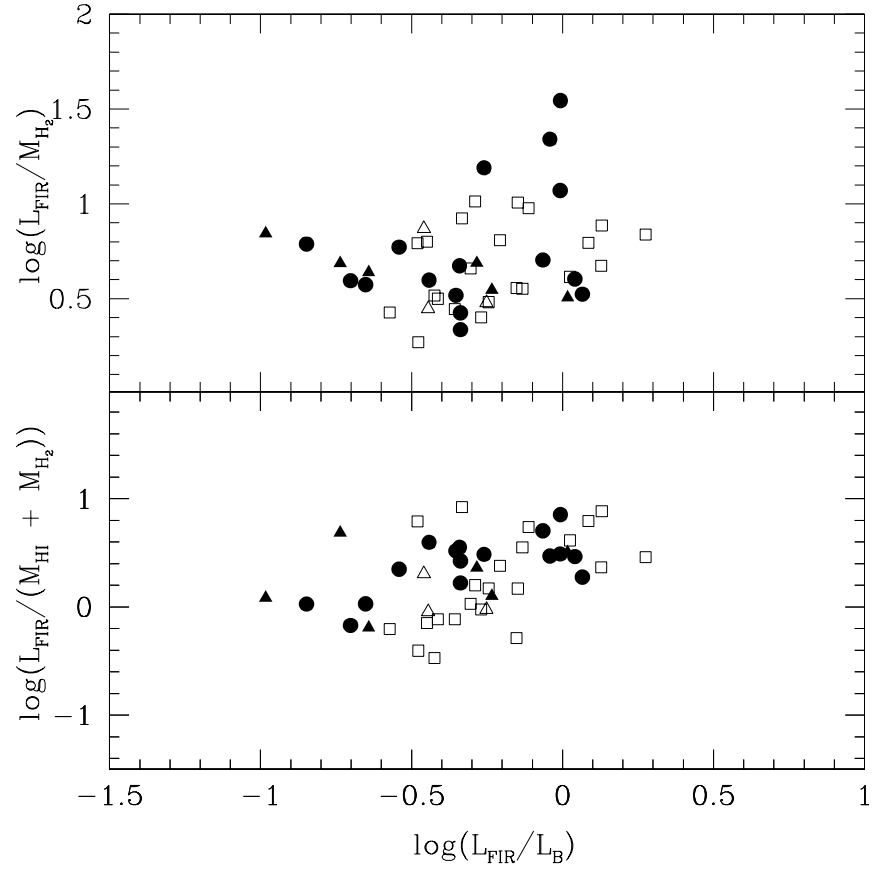

Fig. 11. Upper panel: FIR luminosity normalized by molecular gas as a function of FIR luminosity normalized by blue luminosity. Lower panel: FIR luminosity normalized by total (neutral and molecular) gas as a function of FIR luminosity normalized by blue luminosity. Symbols are the same as in Fig. 4. Luminosities are in $L_{\odot}$ and mass in $M_{\odot}$.

galaxies (ESO-LV3520530, ESO-LV4050180, and ESOLV5480310 - marked in Fig. 12) which were not among the efficient ones, now have higher values. This could be due to the low amount of $\mathrm{HI}$ in these earlier type spirals. If we remove them from our sample and keep a more homogeneous sample in terms of morphology, we find that $\log \left(M_{H I} / M_{H_{2}}\right)$ is higher for the HDS than for the CS. The cumulative distribution function (Fig. 13) of the HDS is significantly different than that of the CS. However, we have HI data for only 12 galaxies of the HDS which are later than $\mathrm{Sb}$, therefore, a larger and homogeneous sample in terms of morphology should be observed in order to confirm this result.

\section{Summary and conclusions}

We have performed a detailed comparison between properties of intermediate Hubble type galaxies in dense environments (HDS) and in the field (CS). By using several different diagnostics of global properties we have found a trend for the gaseous content and star formation properties of the high and low density samples. Intermediate Hubble type galaxies in dense environments have, on average:

- Lower gas content than field galaxies (i.e. lower $M_{\text {gas }} / L_{\mathrm{B}}$ ratio);

- Higher atomic gas fraction than field galaxies (i.e. a higher $M_{\mathrm{HI}} / M_{\mathrm{H}_{2}}$ ratio); 


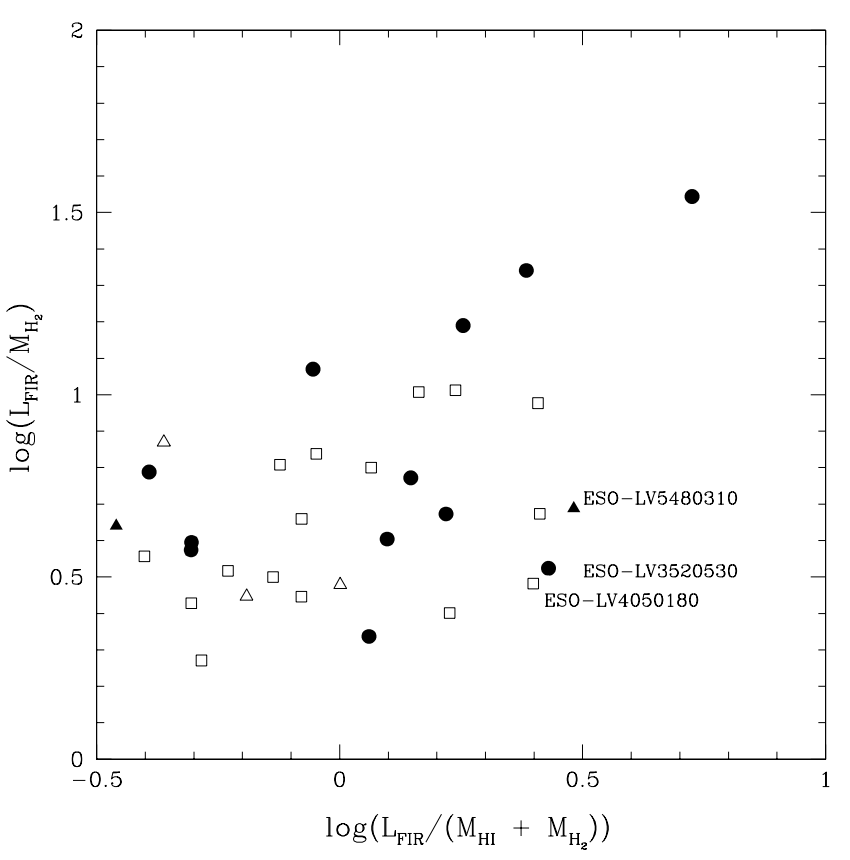

Fig. 12. FIR luminosity normalized by molecular gas as a function of FIR luminosity normalized by total (atomic and molecular) gas. Symbols are the same as in Fig. 4. Luminosities are in $L_{\odot}$ and mass in $M_{\odot}$.

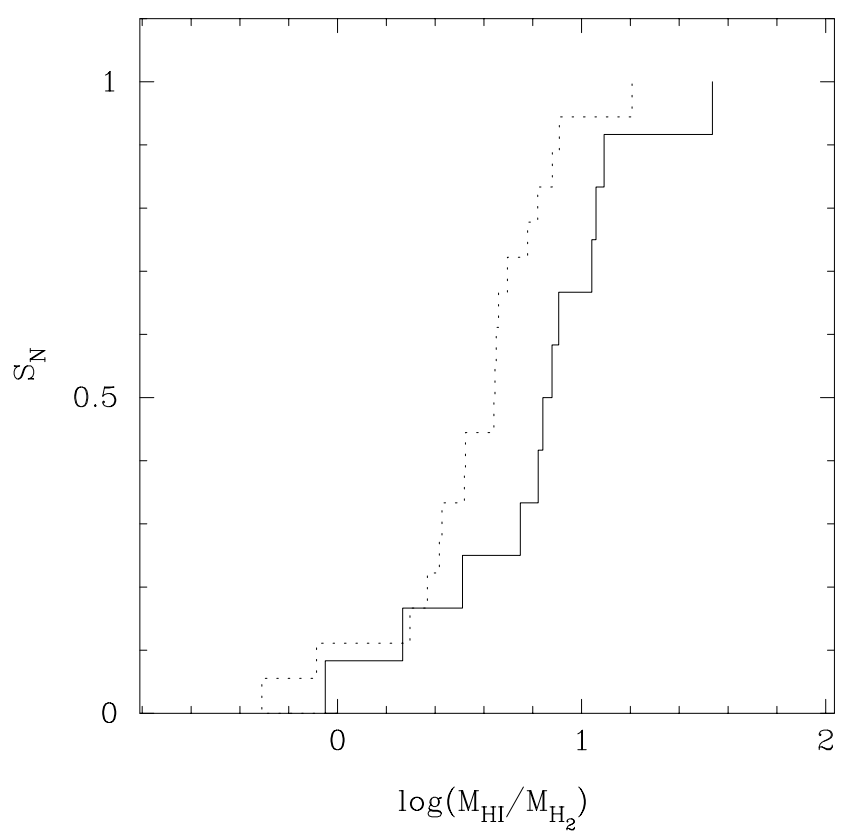

Fig. 13. Cumulative probability distribution of atomic and molecular gas ratio. HDS is marked by solid line and CS by dotted line.

- Lower current star formation rate than field galaxies (i.e. lower $L_{\mathrm{FIR}} / L_{\mathrm{B}}$ ratio);

- The same star formation efficiency as field galaxies (i.e. the same $L_{\mathrm{FIR}} / M_{\mathrm{H}_{2}}$ or $L_{\mathrm{FIR}} / M_{\text {gas }}$ ratio).

Although none of the above results stand out as a single strong diagnostic given their statistical significance (see Table 4), taken together they suggest a trend for diminished gas content and star formation activity in galaxies in high density environments.

What can be the physical processes behind this result? It has long been believed that gravitational interaction is a sufficient condition for transporting gas from the outer regions of galaxies to the inner regions, and thereby increasing the star formation activity. If this is the case for our high density sample, the lower gas content could be the result of gas exhaustion through an increased star formation activity in the past. However, it seems unlikely that we should have selected only those systems which experienced enhanced star formation rates in the past. An alternative and more likely interpretation is that repeated close encounters, experienced by galaxies in dense environments, removes gas from the galaxies as well as leads to an inhibition of the formation of molecular gas from the atomic phase. The similarities in star formation efficiency then suggest that the physical processes controling the formation of stars from the molecular gas are local rather than global.

In addition, we find that $6(38 \%)$ of the HDS galaxies and $3(14 \%)$ of the CS galaxies are classified as LINERS. These are very limited numbers but a few interesting trends among the LINERS are suggested by our data. First of all, the LINERS show a very good correlation between molecular gas and $L_{\mathrm{FIR}}$, they have a lower dust temperature than the non-LINER HDS and CS galaxies and they have a lower average star formation efficiency. These findings are consistent with the LINERS as aging starbursts rather than being powered by an AGN. A larger sample is needed to study these correlations in more detail.

Acknowledgements. We thank the ON team of observers at the ESO1.52m, in particular Christopher Willmer for helping with the data reduction. Henrique Schmitt is thanked for valuable suggestions regarding the stellar contamination as well as the anonymous referee for valuable suggestions which helped improving our paper. This research has made use of the NASA/IPAC Extragalactic Database (NED) which is operated by the Jet Propulsion Laboratory, California Institute of Technology, under contract with the National Aeronautics and Space Administration. D.F.M. was supported partially by CNPq Fellowship 301456/95-0, and the Swedish Vetenskapsrådet (NFR) project number F620-489/2000. M.A.G.M. was supported by CNPq grant 301366/86-1. T.W. was supported by Vetenskapsrådet project number F1299/1999.

\section{References}

Aaronson, M., Huchra, J., Mould, J., Schechter, P. L., \& Tully, R. B. 1982, ApJ, 258, 64

Alonso-Herrero, A., Rieke, M. J., Rieke, G. H., \& Shields, J. C. 2000, ApJ, 530, 688

Allam, S., Assendorp, R., Longo, G., Braun, M., \& Richter, G. 1996, A\&AS, 117, 39

Baldwin, J. A., Phillips, M. M., \& Terlevich, R. 1981, PASP, 93,5 
Barton, E. J., Geller, M. J., \& Kenyon, S. J. 2000, ApJ, 530, 660

Boselli, A., Mendes de Oliveira, C., Balkowski, C., Cayatte, V., \& Casoli, F. 1996, A\&A, 314, 738

Braine, J., Combes, F., Casoli, F., et al. 1993, A\&AS, 97, 88

Bushouse, H. A. 1987, ApJ, 320, 49

Casoli, F., Boisse, P., Combes, F., \& Dupraz, C. 1991, A\&A, 249,359

Casoli, F., Dickey, J., Kazes, I., et al. 1996, A\&A 309, 43

Combes, F., Prugniel, P., Rampazzo, R., \& Sulentic, J. W. 1994, A\&A, 281, 725

Coziol, R., Iovino, A., \& de Carvalho, R. R. 2000, AJ, 120, 47

Dahari, O. 1984, AJ, 89, 966

de Mello, D. F., Maia, M. A. G., \& Wiklind, T. 2002, A\&A, 381, 761 (Paper I)

de Vaucouleurs, G., de Vaucouleurs, A., Corwin, H. G. Jr., et al. 1991, Third Reference Catalogue of Bright Galaxies (RC3) (New York: Springer)

Gerin, M., \& Casoli, F. 1994, A\&A, 290, 49

Hashimoto, Y., Oemler, A. Jr., Lin, H., \& Tucker, D. L. 1998, ApJ, 499, 589

Hickson, P. 1997, ARA\&A, 35, 357

Hildebrand, R. H. 1983, QJRAS, 24, 267

Ho, L. C., Filippenko, A. V., \& Sargent, W. L. W. 1997, ApJS, 112,315

Horellou, C., Casoli, F., Combes, F., \& Dupraz, C. 1995, A\&A, 298,743

Horellou, C., \& Booth, R. 1997, A\&AS, 126, 3

Huchtmeier, W. K. 1997, A\&A, 325, 473

Ji, L., Chen, Y., Huang, J. H., Gu, Q. S., \& Lei, S. J. 2000, A\&A, 355, 922

Keel, W. C., Kennicutt, R. C. Jr., van der Hulst, J. K., \& Hummel, E. 1985, AJ, 90, 708

Keel, W. C. 1996, AJ, 111, 696

Kenney, J. D. P., \& Young, J. S. 1988, ApJ, 326, 588

Kennicutt, R. C. Jr. 1983, ApJ, 272, 54

Kennicutt, R. C. Jr. 1998, ARA\&A, 36, 189

Kennicutt, R. C. Jr., Bothun, G. D., \& Schommer, R. A. 1984, AJ, 89, 1279
Kennicutt, R. C. Jr., \& Keel, W. C. 1984, ApJ, 279, 5

Lauberts, A., \& Valentijn, E. A. 1989, The Surphace Photometry Catalogue of the ESO-Uppsala Galaxies (Garching bei München: ESO)

Larson, R. B., \& Tinsley, B. M. 1978, ApJ, 219, 46

Leon, S., Combes, F., \& Menon, T. K. 1998, A\&A, 330, 37

Liu, C. T., \& Kennicutt, R. C. Jr. 1995, ApJ, 450, 547

Maia, M. A. G., da Costa, L. N., \& Latham, D. W. 1989, ApJS, 69,809

Maia, M. A. G., Willmer, C. N. A., \& da Costa, L. N. 1998, AJ, 115, 49

Maiolino, R., Ruiz, M., Rieke, G. H., \& Papadopoulos, P. 1997, ApJ, 485, 552

Mendes de Oliveira, C., \& Hickson, P. 1994, ApJ, 427, 684

Mihos, J. C., \& Hernquist, L. 1994, ApJ, 437, L47

Moshir, M., Kopar, G., Govrow, T., et al. 1990, IRAS Faint Source Catalogue, version 2.0.

Pastoriza, M. G., Bica, E., Maia, M. A. G., \& Dottori, H. 1994, ApJ, 432, 128

Press, W. H., Flannery, B. P., Teukolsky, S. A., \& Vetterling, W. T. 1989, Numerical Recipes, The Art of Scientific Computing (FORTRAN Version) (Cambridge, University Press)

Roberts, M. S., \& Haynes, M. P. 1994, ARA\&A, 32, 115

Sage, L. J. 1993, A\&A, 272, 123

Sanders, D. B., Scoville, N. Z., \& Soifer, B. T. 1991, ApJ, 370, 158

Sulentic, J. W., \& de Mello Rabaça, D. F. 1993, ApJ, 410, 520

Szomoru, A., van Gorkom, J. H., Gregg, M. D., \& Strauss, M. A. 1996, AJ, 111, 2150

Thronson, H. A., \& Telesco, C. M. 1986, ApJ, 311, 98

Veilleux, S., \& Osterbrock, D. E. 1987, ApJS, 63, 295

Young, J. S., \& Knezek, P. 1989, ApJ, 347, L55

Young, J. S., Shuding, X., Kenney, J. D. P., et al. 1989, ApJS, 70,699

Wiklind, T., Combes, F., \& Henkel, C. 1995, A\&A, 297, 643

Williams, B. A., \& Rood, H. J. 1987, ApJS, 63, 265 